\title{
Multiple tender, deep nodules on the legs of a seven-year-old boy
}

\author{
Christopher Dalby
}

CASE

A boy, aged seven years, presented with both parents concerned about the development over the past two days of bruise-like skin markings over his anterior shins (Figures 1, 2). The skin changes were ill-defined, indurated and tender, and the patient found it uncomfortable to walk. There was no clear cause for these skin changes; the boy and his parents specifically denied any trauma. There was no evidence of any other bruising or injury. These skin markings arose in the context of the boy having presented a week earlier with fevers and a sore throat. At that time, a diagnosis of upper respiratory tract infection was made.

He had been otherwise well and there was no other significant medical history.

\section{Question 1}

What is the most likely diagnosis?

\section{Answer 1}

This patient's symptoms are consistent with erythema nodosum, which is the most common form of panniculitis. Erythema nodosum is an inflammatory condition of subcutaneous fat that presents with inflamed nodules or plaques. ${ }^{1,2}$

\section{Question 2}

How does the diagnosed condition typically present?

\section{Answer 2}

The lesions of erythema nodosum typically appear acutely and symmetrically on the

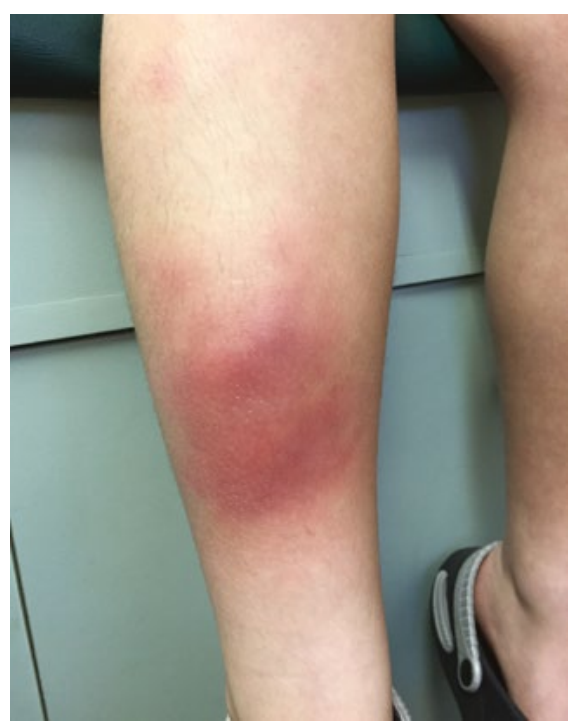

Figure 1. Right leg

anterior shins, knees and ankles, but may occur at other body sites. ${ }^{1,3}$ The initial lesions are $1-5 \mathrm{~cm}$ in diameter. ${ }^{1,3}$ They may be preceded by fatigue, low-grade fever, malaise and joint pain. ${ }^{1,3}$ As erythema nodosum progresses, the lesions can merge and take on a bruise-like appearance. ${ }^{1,3}$

\section{Question 3}

What age group and demographic does the diagnosed condition generally affect?

\section{Answer 3}

Erythema nodosum can affect any age group, but most commonly affects those aged $20-40$ years, with a bias of $6: 1$ towards women. ${ }^{2}$

\section{Question 4}

What is thought to be the pathogenesis of the diagnosed condition? What are the possible causes?

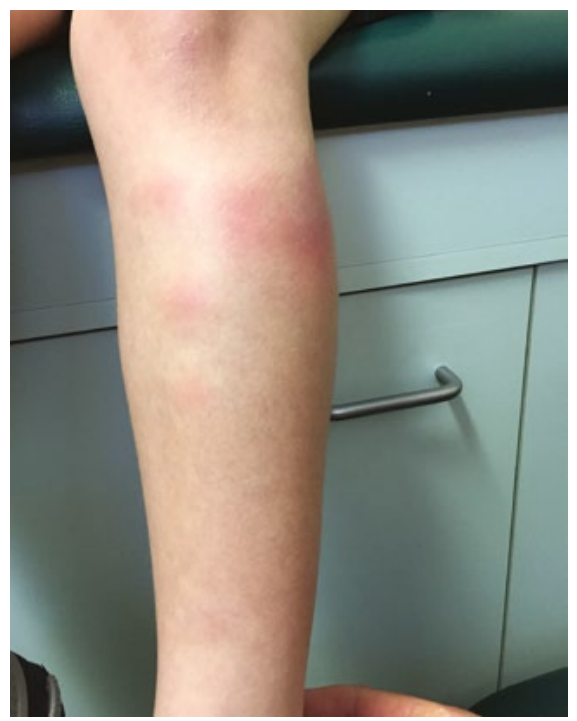

Figure 2. Left leg

\section{Answer 4}

Erythema nodosum is generally considered to be a type IV delayed hypersensitivity reaction, resulting from the deposition of immune complexes in the subcutaneous fat. ${ }^{1-3}$ However, the exact mechanism is not fully understood..$^{1-3}$ There is a range of potential triggers, with post-streptococcal infection the common identifiable cause. ${ }^{1-3}$ Other aetiologies include a range of bacterial, viral and fungal infections, medications (notably including the oral contraceptive), chronic inflammatory conditions such as sarcoidosis and inflammatory bowel disease, pregnancy and, rarely, malignancy. ${ }^{1-3}$ However, half of all cases of erythema nodosum have no identifiable cause found. ${ }^{1-3}$

\section{Question 5}

What are other differential diagnoses that could be considered? 


\section{Answer 5}

This patient's symptoms are most consistent with erythema nodosum, but differential diagnoses to consider include:

- non-accidental injury

- subcutaneous infections ${ }^{2}$

- superficial thrombophlebitis ${ }^{2}$

- other disorders of fatty tissue (eg lipoid dystrophies, nodular fat necrosis, necrobiosis lipoidica) ${ }^{3}$

- systemic disease such as scleroderma ${ }^{3}$

- lupus erythematosus profundus ${ }^{3}$

- scleroderma neonatorum ${ }^{3}$

- granulomas. $^{3}$

\section{Question 6}

What diagnostic tests need to be considered to confirm the diagnosis?

\section{Answer 6}

Erythema nodosum is considered to be a clinical diagnosis, and biopsy is generally not indicated. ${ }^{1-3}$ Biopsy should only be considered for unusual cases. ${ }^{1-3}$

When the diagnosis is uncertain, investigations for infective processes should be considered. ${ }^{1}$ This may include throat bacterial culture, antistreptolysin O titre for Group A Streptococcus, and chest X-ray. ${ }^{1}$

\section{Question 7}

What management is required for this condition?

\section{Answer 7}

As the lesions typically resolve spontaneously in 2-6 weeks without scarring, ${ }^{1,2}$ treatment should be focused on symptomatic support and removal of the causative agent if there is one (eg cessation of the oral contraceptive if this is thought to be cause).$^{1-3}$ Rest, elevation and compression may relieve symptoms. ${ }^{1-3}$ Anti-inflammatory agents, such as ibuprofen, have also been shown to be of benefit. ${ }^{1-3}$

\section{CASE CONTINUED}

The day after this presentation, the boy was unable to walk because of the pain in his legs. Because of this, his parents took him to the nearby tertiary paediatric hospital. During the subsequent threeday admission, his admitting team confirmed the diagnosis of erythema nodosum. After discharge, his condition resolved without complication.

\section{Key points}

- The most common recognised cause of erythema nodosum is streptococcal infection.

- Erythema nodosum is primarily a clinical diagnosis, although biopsy may be warranted in atypical cases.

- Management is supportive as the condition resolves spontaneously.

\section{Author}

Christopher Dalby DCH, BPharm, MBBS, FRACGP, Associate Lecturer, University of Queensland, Qld. c.dalby@uq.edu.au

Competing interests: None.

Provenance and peer review: Not commissioned, externally peer reviewed.

\section{References}

1. Blake T, Manahan M, Rodins K. Erythema nodosum - A review of an uncommon panniculitis. Dermatol Online J 2014;20(4):22376.

2. Gilchrist $\mathrm{H}$, Patterson JW. Erythema nodosum and erythema induratum (nodular vasculitis): Diagnosis and management. Dermatol Ther 2010;23(4):32027. doi: 10.1111/j.1529-8019.2010.01332.x.

3. Schwartz RA, Nervi SJ. Erythema nodosum: A sign of systemic disease. Am Fam Physician 2007;75(5):695-700.

correspondence ajgp@racgp.org.au 